\title{
Addictive agents and intracranial stimulation (ICS): Pressing for ICS under the influence of ethanol before and after physical dependence
}

\author{
DEBRA J. MAGNUSON and LARRY D. REID \\ Bradley University, Peoria, Illinois 61606
}

\begin{abstract}
Rats were fixed with chronically indwelling electrodes for intracranial stimulation (ICS) of the lateral hypothalamus. Rats pressed daily, in brief sessions, for the ICS. Twenty-five minutes prior to sessions with ICS, rats were given injections of .5 and $1.5 \mathrm{~g} / \mathrm{kg}$ of ethanol. The smaller dose produced a slight increase in pressing, whereas the larger dose produced a large decrease in pressing. Then one group of rats was made physically dependent on ethanol by force feeding them every few hours across 4 days. The other group was fed only saline. Seven days after the procedure establishing dependence, rats were again tested for effects of ethanol injections on pressing for ICS. After physical dependence, large doses of ethanol did not produce as great a depression in pressing for ICS.
\end{abstract}

St. Laurent (1972) and Flanagan and Reid (in press) did not observe marked acceleration in pressing for intracranial stimulation (ICS) with ethanol injections. At times of peak blood ethanol levels (about 25 min after injections), doses of $.5 \mathrm{~g} / \mathrm{kg}$ produced a slight acceleration of pressing; a smaller dose $(.25 \mathrm{~g} / \mathrm{kg})$ produced no effect, and larger doses $(1.0 \mathrm{~g} / \mathrm{kg}$ or greater $)$ depressed rates of pressing. Kelley, Tadeusik, Magnusion, and Reid (Note 1) found that subsequent to the ingestion of considerable quantities of ethanol by rats, injections of $1.0 \mathrm{~g} / \mathrm{kg}$ of ethanol tended to produce acceleration of pressing for ICS and larger doses resulted in less depression of responding. Those results led to speculation that the processes following assimilation of large quantities of ethanol led to increased pressing under ethanol injections, which in turn may reflect increased potential for positive reinforcement from ethanol.

The purpose of this study was to determine if the processes of physical dependence would change responsiveness to ICS. A technique described by Majchrowicz (1975) was used to induce physical dependence. This technique involved maintaining high blood ethanol levels over a 4-day period. Rats were tested for the effects of ethanol injections on pressing for ICS before and after physical dependence and concomitant withdrawal symptoms.

\section{METHOD}

\section{Subjects}

Each of 23 adult male Sprague-Dawley rats, weighing 200 to

This research was supported by Grant DA01049, National Institute on Drug Abuse, DHEW, and a grant from the Distilled Spirits Council of the United States. D. Magnuson is now at the Department of Anatomy, University of Illinois at the Medical Center, Chicago, Illinois. L. Reid is now at the Department of Psychology, Rensselaer Polytechnic Institute, Troy, New York 12181.
$250 \mathrm{~g}$, was fixed with a chronically indwelling bipolar electrode. The two stainless steel electrode wires were insulated except at the cross-section of their tips and separated only by their insulation. The tips were aimed for the lateral hypothalamus, a site for which ICS is positively reinforcing. Rats were housed individually with food and water always available.

Of the initial 23 subjects, 4 died during the procedures producing dependence, most likely due to the combined depressant effects of ethanol and slight respiratory infections. Two later died from unknown causes. One subject appeared to have suffered some cerebral damage manifested by hyperactivity. Analyses of the data were then done on the scores of the remaining 16 rats. This mortality rate is characteristic of the procedures for inducing physical dependence on ethanol in rats (Majchrowicz, 1975), and was planned for in this study.

\section{Apparatus}

Rats were tested for pressing in an experimental chamber fitted with a lever, and housed in a lighted sound-attenuating box. Depression of the lever resulted in a single pulse of ICS of $60-\mathrm{Hz}$ sine waves of $.25 \mathrm{sec}$, the intensity of which varied but was always less than 50 microA. ICS was delivered by way of light, flexible leads attached to a slip-ring assembly, allowing the rats unhampered movement. The apparatus was the same as that of Flanagan and Reid (in press) and Kelley et al. (Note 1).

\section{Procedure}

In general, the procedures for testing the effects of ethanol injections on pressing for ICS were similar to those of Flanagan and Reid (in press). After at least 7 days of recovery from surgery, subjects were tested for barpressing for ICS. Subsequently, rats were run at four intensities $(5,10,20 \mathrm{micro} A$, and a higher intensity which produced rapid press rates, 30 to 45 microA) for 2-min periods at each intensity. Press rates were automatically recorded. Before each 2-min measurement period, each rat was allowed to press for that intensity at least 10 times. If the subject did not press at that intensity, 10 ICSs were delivered by the experimenter while shaping the animal to press. Since the lower intensities may have been below threshold for reinforcement, some subjects hardly pressed for those intensities. All rats, however, showed sustained pressing at 20 microA and the selected higher intensity. Rats pressed under this daily regimen of tests for the four intensities until press rates stabilized (10 days).

To assess the effect of ethanol on pressing, daily testing 
continued while a procedure of ethanol injections was instituted. Doses of ethanol of .5 and $1.5 \mathrm{~g} / \mathrm{kg}$ were administered $25 \mathrm{~min}$ prior to testing for ICS press rates. The procedure spanned four daily sessions for each of the two doses of ethanol: (1) a day of baseline, (2) a day of pre-ethanol placebo, (3) a day of ethanol, and (4) another day of baseline. Rats were randomly assigned to one of two groups: One group received a $.5-\mathrm{g} / \mathrm{kg}$ dose of ethanol first, while the other group received a $1.5 \mathrm{-g} / \mathrm{kg}$ dose first. At the end of testing with the second dose, another day of baseline was run. The ethanol injections were $10 \%$ ethanol in physiological saline (weight/volume) given intraperitoneally. Placebo injections were saline solutions of the same volume as the corresponding ethanol injections.

Following stabilization of press rates and tests with ethanol injections, subjects were assigned to one of two groups such that the resultant groups did not differ in reactivity to ethanol with respect to changes in press rates for ICS with ethanol injections. One group of eight subjects (the ethanol-dependent group) received, over the next 4 days, $9-15 \mathrm{~g}$ of pure ethanol/kilogram/ day in a $20 \%$ ethanol solution (weight/volume) in three to five doses per day, according to the intragastric procedure of Majchrowicz (1975). The exact dosing regimen was determined for each rat based on behavioral observations of the degree of loss of righting reflex, gait, muscle tone, and other factors. Depending on the stage of intoxication indexed by the behavioral measures, the rats were given an amount of ethanol sufficient to maintain intoxication with high blood ethanol levels until the next dosing. The other group of eight subjects received tap water in equal amounts.

To control for the effects of weight loss of the dependent group on subsequent testing, the control group was food deprived. The control group lost about the same amount of body weight, that is, $20 \%$ of pretreatment weight, as the dependent group during the period of ethanol dosing and withdrawal. Both groups received daily $.5 \mathrm{ml}$ of a multivitamin and mineral supplement (ViDaylin-M). Subsequent to the 4-day period of intubations, the rats were monitored for signs of withdrawal for 2 to 3 days.

Seven days were allowed to lapse from the last intubation to the beginning of the second testing regimen. The second testing regimen with ICS was the same as the procedures of the first testing, except that these tests were preceded by 1 day of baseline.

Subsequent to tests with ICS, the subjects were sacrificed with an overdose of Nembutal. They were then intracardially perfused with saline followed by a Formalin solution. The brains were removed, frozen, and sliced. These slices, about 75-90 microns in thickness, were then treated as photographic negatives, and photographs of them were taken to aid in determination of the site of ICS.

\section{RESULTS}

The procedures of Majchrowicz (1975) to induce physical dependency succeeded. With termination of ethanol intubations, the rats showed rapid weight loss, propensity to seizures, hypersensitivity to being handled, tremors, and a sick appearance. These symptoms were not observed on the third day after termination of giving alcohol.

The rats' pressing for ICS during placebo trials was not discernibly modified by the treatment procedures, nor was there any apparent interaction between the kind of treatments (ethanol and placebo intubations) and pressing for ICS under placebo before and after treatments.
An inspection of the data suggested no differential effects of the procedures on pressing with respect to the different intensities of ICS, that is, if a rat's pressing was affected, pressing at all intensities was affected in a similar fashion. Because of this apparent lack of effect and because some rats did not press for the lower intensities, the number of presses was summed across intensities and this sum became a rat's score for a particular day's test. Also, it seemed to matter little whether or not the rats got a $.5 \mathrm{-g} / \mathrm{kg}$ dose first or second, in the series of tests; therefore, the potential order effect was ignored in subsequent analyses.

To assess the effects of ethanol, while taking into account each rat's level of pressing, the score under placebo was subtracted from the comparable score under ethanol. A positive score, therefore, indicates increased pressing due to ethanol, while a negative score indicates a decrease in pressing. With these reductions in the data, there was an array of difference scores conforming to a 2 by 2 by 2 matrix having repeated measures with factors related to the two groups (dependent and controls), the two tests (one before and one after intubation), and the two doses.

An analysis of variance (ANOVA) on the scores of the repeated measures yielded for the factor of Dose an $\mathrm{F}(1,14)=28.61, \mathrm{p}<.01$; for the factor of Group by Before-After treatments an $F(1,14)=6.41, p<.05$; for the factor of Dose by Before-After an $F(1,14)=15.41$, $\mathrm{p}<.01$. No other sources of variance were reliable (ps>.50).

Figure 1 presents the data of the reliable dose relationship. With pressing for ICS under the $.5-\mathrm{g} / \mathrm{kg}$ dose, there was a slight, although not reliable, increase in pressing over the placebo scores on the first tests $[\mathrm{t}(14)=1.74, \mathrm{p}<.15]$. The $1.5 \mathrm{-g} / \mathrm{kg}$ dose produced $\mathrm{a}$ large significant decrease in pressing $[\mathrm{t}(14)=-5.33$, $\mathrm{p}<.01]$.

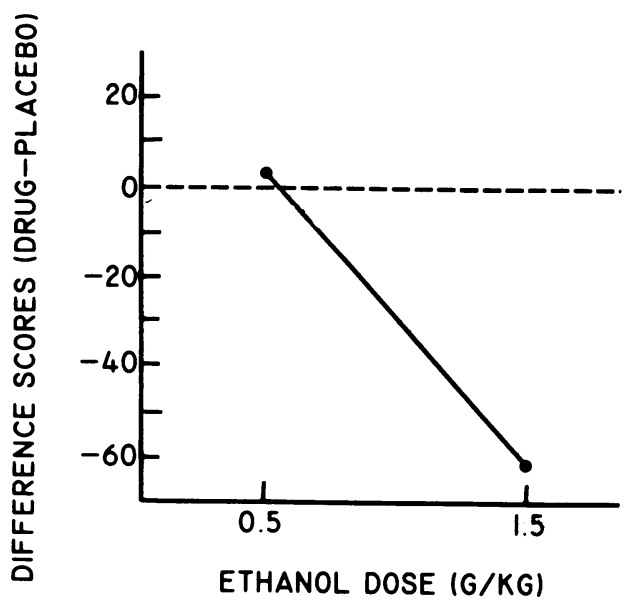

Figure 1. The mean effects of two doses of ethanol on pressing for ICS. The values are mean difference scores across all conditions of testing. 


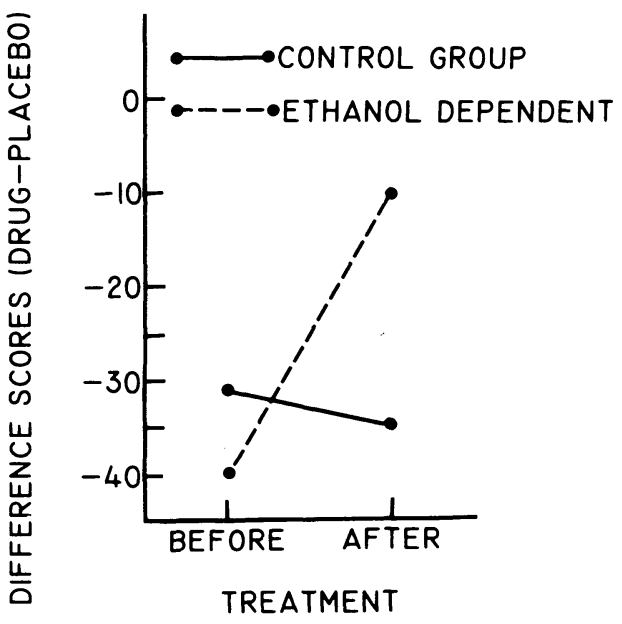

Figure 2. The mean effects of ethanol across both doses on pressing for ICS before and after treatments. The treatment for one group was procedures to induce physical dependency on alcohol.

A summary of the Group by Before-After interaction is shown in Figure 2. The dependent rats' pressing was less depressed under ethanol after the dependency $t(7)=3.38, p<.02]$. The control rats' pressing did not change reliably across the tests before and after treatments.

Across all rats, the mean difference score for the .5 $\mathrm{g} / \mathrm{kg}$ dose was 14.8 prior to treatments and -.90 after treatments, $[\mathrm{t}(15)=2.4, \mathrm{p}<.05]$. The mean difference for the $1.5 \mathrm{~g} / \mathrm{kg}$ was -85.0 prior to treatments and -37.1 after treatments $[t(15)=-3.8, p<.01]$. The dependency of the one group and the food deprivation of the control group or the initial dosing with ethanol evidently somehow changed the rats' reactivity to ethanol.

Examination of brain slices indicated that all sites of ICS were in the lateral hypothalamic area with electrode tips in the medial forebrain bundle and the zona incerta.

\section{DISCUSSION}

The procedure producing physical dependence had remarkably little effect on pressing for ICS when rats were not challenged with ethanol. When tested for pressing for ICS under ethanol, it was found that the $1.5 \mathrm{~g} / \mathrm{kg}$ produced less suppression of pressing than did the same dose prior to physical dependence.

Both the control and the dependent group pressed less under the $.5-\mathrm{g} / \mathrm{kg}$ dose of ethanol after treatments, but the dose did not lead to differential effects for the two groups. It is of interest to note that the extensive dosing with ethanol did not lead to increased pressing with the small dose of ethanol. The extensive dosing did, however, reduce the suppression under the larger dose. These results parallel our results, in similar testing paradigms, with morphine as the addictive agent. It is the suppressive effects of addictive agents that shows tolerance.

There were wide individual differences in reactivity to ethanol injections; some rats' pressing was accelerated while under doses of ethanol but others' pressing was markedly depressed. We attempted to correlate site of ICS with the effects of ethanol injections but could discern no clear pattern of results. Perhaps, however, rats with electrodes in the zona incerta were more apt to increase their pressing with ethanol injections.

\section{REFERENCE NOTE}

1. Kelley, K. L., Tadeusik, C. J.. Magnuson, D. J., \& Reid, L. D. Addictive agents and intracranial stimulation [ICS]: Pressing for ICS under the influence of ethanol before and after forced ethanol consumption. In preparation.

\section{REFERENCES}

Flanagan, R. W., \& Reid, L. D. Addictive agents and intracranial stimulation: Ethanol and self-stimulation. Journal of Studies on Alcohol, in press.

MajCHRowicz. E. Induction of physical dependence upon ethanol and the associated behavioral changes in rats. Psychopharmacologia (Berlin), 1975, 43, 245-254.

St. LAURENT. J. Brain centers of reinforcement and the effects of alcohol. In B. Kissin \& H. Begleiter (Eds.), The biology of alcoholism: Phvsiology and behavior (Vol. 2). New York: Plenum Press, 1972.

(Received for publication June 28, 1977.) 